Volume 9, No.4, July - August 2020

International Journal of Advanced Trends in Computer Science and Engineering

Available Online at http://www.warse.org/IJATCSE/static/pdf/file/ijatcse289942020.pdf

https://doi.org/10.30534/ijatcse/2020/289942020

\title{
Using Neural Network for Simulations to Improve the Quality of Disease Diagnosis: Technical Aspects
}

\author{
Denis V. Gilev ${ }^{1}$, Oleg V. Loginovsky ${ }^{2}$ \\ ${ }^{1}$ Ural Federal University named First President of the Russian Federation Boris Yeltsin, Russia, \\ deni-gilev@narod.ru \\ ${ }^{2}$ South Ural state University, Russia, deni-gilev@ narod.ru
}

\begin{abstract}
Mathematical models are important for the processes of cognition and decision-making. They provide a concise representation of significant relationships in the description of objects and situations. Adding new relationships leads to narrowing the scope of applicability of the model. The formula is an example of a compressed description of a potentially infinite set of objects and situations.

Knowledge processing is based on the use of mathematical methods. In this case, it is the most thorough, at least from the point of view of strict logic and consistent formalization. To process knowledge, we must present it in some form that is convenient for analysis. Thus, when analyzing data and knowledge, we do not use them directly, but their representations. Mathematical models of objects and phenomena are an effective way of representation. This is now the most powerful method of cognition of processes, objects and phenomena. Modeling is a special way of scientific research. A mathematical model of an object is a mathematical structure interpreted within a given domain.
\end{abstract}

Keywords : neural network, simulations, improve, quality.

\section{INTRODUCTION}

The need to use mathematical models is determined by the following considerations [1-5]:

- in many cases, direct experiments on objects are not possible; at the same time, experiments on models are possible;

- models allow you to simulate the consequences of various scenarios of system behavior and make predictions [6].

Modeling makes it possible to get to the bottom of latent factors and phenomena that are not directly observed. Moreover, informal factors can also be modeled [7]. As examples, we can use factor analysis and pattern recognition in modeling decision-making factors, for example, in identifying the root causes of deviations in people's behavior in the socio-economic sphere from rational behavior. Creating a model is the most difficult stage of research, and analyzing it (since it is much simpler than the object being modeled) is the least time - consuming part. The" incomprehensible efficiency " of mathematics is partly explained by the greater simplicity of the model than the modeled object [8].

The model is a tool for effective evaluation of management strategies, and the method for the problem of choosing solutions depends on the characteristics of the model.

Conventionally, the methods of scientific research can be divided into theoretical and empirical. At the same time, the modeling method has its own special place and features. When a modeling method is used, the model acts as an analog and substitute for the object [9].

A mathematical model is a set of certain mathematical relations - equations and inequalities that connect variables and parameters, systems of inclusions, iterative processes, and software systems. These relationships describe some aspects of the object, phenomenon, or process being studied. Models can also take the form of neural networks. The modeling method consists of studying the object on the material of the relations describing it [10]. The results of model research are transferred to the object (but not directly, because the object and its model are still not the same thing).

\section{METHODOLOGY}

The model can be a software system, a neural network, or an expert system. A neural network can take the form of either a computer program or - better yet - a neurocomputer. The computer itself, with its rich programming content, is the best known model. Any person may in certain respects be better than his computer in the intellectual sense [1-4].

Experiments and mathematical models have always been the main tools in the natural Sciences. But these concepts have long been important also in Economics, sociology, psychology, and, most importantly, in medicine [5-9].

The degree of complexity of the model should be specifically chosen and justified, it should be adequate for the purposes of modeling.

The mathematic model is an $n$-dimensional vector

$x=\left[x_{1}, \ldots, x_{n}\right]$.

Parameters whose values are numbers $x_{1}, \ldots, x_{n}$ are called object attributes. To characterize objects, you can choose different feature systems, and the task of evaluating information content involves finding the optimal (in terms of one or another optimality criterion) feature system. In General, the problem is divided into subtasks: selecting useful 
features from their initial list, suggested by experts, converting features, and evaluating the usefulness of subsystems of features [10].

The selection of useful traits can be formalized as the task of maximization of detection quality, provided that the cost of measuring characteristics fit into a specific budget or minimization of cost for a given quality of recognition [11-15].

One of the most important tasks of feature transformation is to construct a so-called straightening transformation, in which the new feature system allows you to divide the precedent sets by hyperplanes [16].

Feature evaluation uses a comparison of the recognition quality when the feature is present with the quality obtained when the feature is excluded [17].

Let's assume that a finite set $X$ in space $R^{n}$ is given, representing the material of observations. This set should be divided into three subsets $Y, Z, V$, where $Y, Z$ consist of elements whose class affiliation is known, but we configure the decisive rule on one set, and control its quality on the other. The elements of a subset $V$ are classified based on the developed decision rules. Elements of sets can be adjusted within acceptable limits to obtain simpler decision rules. In our study, the main issue is the modeling of informal knowledge. The key concepts in this process are diagnostics and case material [18-23].

So, in General, any two objects can be models of each other. However, we only select some objects as suitable models. This selection is based on identifying relevant properties that link objects together.

When constructing mathematical models of image recognition, one of the main initial concepts is the concept of a class (previously classes were often called "images", but it is clear that the term "image" in this case is very unfortunate, because it is not only about visual images). Here, a class is simply a set of objects that are similar to each other in a certain way.

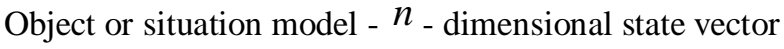
$x=\left[x_{1}, \ldots, x_{n}\right]$,

where ${ }^{x_{i}}$ is the value $i$ of the attribute ( $i$ the parameter measured on the object).

The state space of the objects ${ }^{n}$-dimensional vector space, the space $R^{n}$. In this space, the real set of object States for any particular task forms a valid set $D$.Further, a valid set is assumed to be divided into classes: $D=U D_{i}$. Objects of the same class are similar to each other in some respects; objects of different classes differ in this respect. In doing so, we assume that we know that there are some classes. But we don't know exactly how they were built. And the information about classes that we actually have is examples of objects from different classes. Therefore, we know the precedent subsets of classes: $A_{i}$ - a subset of the class known to us $D_{i}$ the subset of the class that we know. In the discriminant analysis task, you need to use this information to create an idea about classes and give an analytical description of them. For example, we have instances of technologies whose effectiveness we know from our manufacturing experience. It is necessary to provide an efficiency forecast for new prospective technologies that have not yet been sufficiently tested in real production [16-22].

It is clear that the problem of discriminant analysis with several classes is reduced to a sequence of problems, each of which is a two-class problem. Therefore, it is sufficient for us to explain how the problem of constructing models of two classes based on their case sets $A$ and is solved $B$. These sets are parts of sets $D_{1}, D_{2}$, into which a valid set $D_{\text {in a }}$ given space $R^{n}$ is divided .

The last problem is solved as follows. Building a function $f(x)$, where $x$ the allowed set runs $D$ through. This function defines the surface defined by the equation $f(x)=0$, with the specified surface separating the sets $A$ and $B$. This means that $f(x)>0 \forall x \in A$ and $f(x)<0 \forall x \in B$. If this problem is solved and the function $\tilde{f}(x)$ is its solution, then we get the diagnostic rule (also known as the classification rule) for any new object whose state vector is, for example, a vector $y=\left[y_{1}, \ldots, y_{n}\right]$. Namely, we assume: ${ }^{y \in D_{1}}$,if $\tilde{f}(y)>0$, and $y \in D_{2}$, if $\tilde{f}(y)<0$. If $\tilde{f}(y)=0$,then $y_{\text {it belongs to the border }}$ between these classes.

The next pattern recognition model is the taxonomy task (automatic classification task, or "no teacher"recognition task). In this problem, we give a (usually finite) set $M n$ of-dimensional vectors. You need to divide the set $M$ into disjoint subsets (taxa), and the objects included in the same taxon must be sufficiently close to each other in terms of a pre-selected proximity criterion, and elements from different taxa are sufficiently distant from each other by the same criterion. In Economics, this is the task of classification, their mass [23].

The next area of pattern recognition problems is the problem of choosing the characteristics of their transformation, is the task of assessment of signs and systems of signs. This is called in General terms: solve the problem of informative features.

\section{RESULTS AND DISCUSSION}

So, mathematical models and methods in applied areas should make significant use of diagnostics, since the systems based on them should work under conditions of significant a priori uncertainty of knowledge about the properties of the external environment. This makes it difficult to construct them only on the basis of a priori data. Thus, it is necessary to create systems that are able to change their parameters or structure based on processing of available current information in the operational mode in order to ensure the fulfillment of targets 
and tasks over time. Such systems are called trainable or adaptive. They are based on the classification of States.

A system is a device or model that displays (usually unambiguously) a set of input stimuli or signals in a set of output signals. Input signals can be called images, and output signals can be called system responses. A recognition (diagnostic) system or classifier is a system capable of classifying a set of images according to some a priori classification [24].

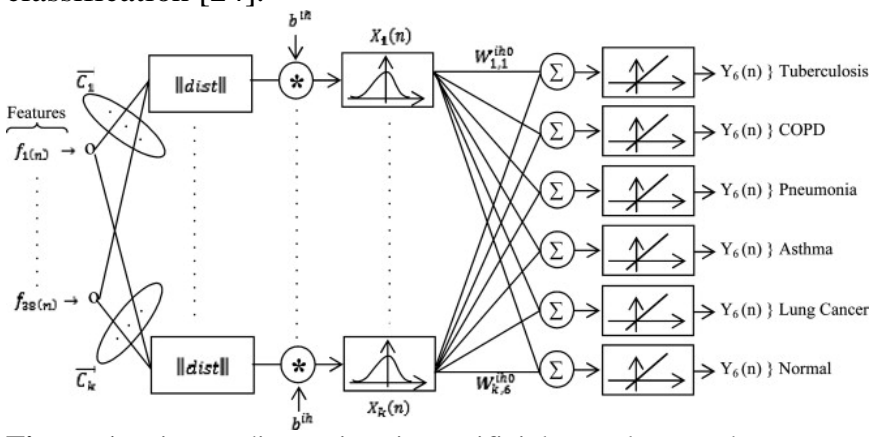

Figure 1: Disease diagnosis using artificial neural network

Figure 1 shows us disease diagnosis in neural network.

Expert data can be some of the attributes used when describing objects and situations in speech recognition [27]. This section discusses the possibilities for predicting how methods will work based on various training data. A crossover algorithm for approximating the optimal number of nearest neighbors is implemented for the nearest neighbor method. We have developed some recommendations for using these methods when solving image recognition problems [25].

Currently, the problems of diagnostics in terms of pattern recognition are becoming more and more practical. It is worth noting that such tasks arise in various fields of human activity, but for us the main thing is in medicine when classifying symptoms and diagnosing diseases [24].

Image recognition is one of the branches of computer science and Cybernetics that deals with solving the problems of concept formation, diagnostics and classification of objects and situations, and evaluating the informative value of factors in various fields of science and technology using methods based on a person's ability to form concepts, recognize and classify objects. The relevance of mathematical support for pattern recognition is constantly increasing [26]. This is due to the need for deeper automation of increasingly complex production methods and their organization, the processing of large flows of information, the need to diagnose and manage flexible technologies, and many other factors.

The main mathematical models for image recognition tasks are as follows:

- discriminant analysis;

- taxonomy;

- assessment and selection of informative feature systems.

The problem of discriminant analysis is as follows: it is required to find functions $x_{1}^{*}, \ldots, x_{k-1} * \in\left\{R^{n} \rightarrow R\right\}$ from some class $X^{*} \in\left\{R^{n} \rightarrow R\right\}$ that separate the data of the set $X_{1}, \ldots, X_{k}$ of spaces $R^{n}$, that is, it is required that for each $j$ $x_{j} *(x)>0, x \in X_{j}, x_{j} *(x) \leq 0, x \in\left(_{i>j} X_{i}, x_{j} * \in X *\right.$ (3) .

The fixed discriminant analysis algorithm matches the set $X_{1}, \ldots, X_{k}, X^{*}$ with the following space partitioning $R^{n}$ : $x$ - as part of the 1 th item, if $x_{1} *(x)>0$; else 2 item, if $x_{2} *(x)>0$; else

...

$(k-1)$ item, if $x_{k-1} *(x)>0$; else

$k$ item, if $x_{1}^{*}(x)>0$,

where $x \in R^{n}$.

Taxonomy problem: find the division of a given finite set $x \in R^{n}$ into subsets

$$
X=\bigcup_{j=1}^{k} X_{j}, X_{i} \cap X_{j}=\varnothing, i \neq j
$$

At the same time, it is necessary that the elements of different subsets $X_{j}$ are sufficiently far from each other, and the elements of the same set are sufficiently close to each other (in terms of the chosen proximity criterion) [28].

One of the exact statements of the taxonomy problem is to find $x \in R^{n}$,and

$$
\begin{aligned}
& \frac{\left|\left(x^{*}, x\right)\right|}{\left\|x^{*}\right\|} \geq \rho, x \in X, \\
& \left|\sum_{x \in X} \operatorname{sign}\left(x^{*}, x\right)\right|<\alpha|X|,
\end{aligned}
$$

where the set $X$ and numbers $\alpha, \rho>0$ are given in

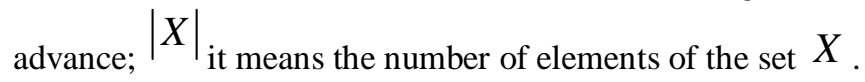

\section{CONCLUSION}

The task of evaluating the information content of feature subsystems is to compare the quality of solving discriminant analysis and taxonomy problems for different feature subsystems (i.e., the coordinates $x_{1}$ of the vector used $\left[x_{1}, \ldots, x_{n}\right]$, which is the model of the object to be recognized).

\section{REFERENCES}

1. Benureau, F. C. Y., and Rougier, N. P. (2017). Re-run, repeat, reproduce, reuse, replicate: transforming code into scientific contributions. Front. Neuroinform. 11:69. doi: 10.3389/fninf.2017.00069

2. Blundell, I., Brette, R., Cleland, T. A., Close, T. G., Coca, D., Davison, A. P., et al. (2018a). Code generation in computational neuroscience: a review of tools and techniques. Front. Neuroinform. 12:68. doi: 10.3389/fninf.2018.00068 
3. Blundell, I., Plotnikov, D., Eppler, J., and Morrison, A. (2018b). Automatically selecting a suitable integration scheme for systems of differential equations in neuron models. Front. Neuroinform. 12:50. doi: 10.3389/fninf.2018.00050

4. Bourque, P., and Fairley, R. E. (2014). SWEBOK: Guide to the Software Engineering Body of Knowledge. Los Alamitos, CA: IEEE Computer Society.

5. Cohen, J. (1988). Statistical Power Analysis for the Behavioral Sciences. New York, NY: Lawrence Erlbaum Associates.

6. Dahmen, W., and Reusken, A. (2005). Numerik für Naturwissenschaftler. Berlin; Heidelberg: Springer.

7. Davison, A., Brüderle, D., Eppler, J., Kremkow, J., Muller, E., Pecevski, D., et al. (2009). Pynn: a common interface for neuronal network simulators. Front. Neuroinform. 2:11. doi: 10.3389/neuro.11.011.2008

8. Furber, S. B., Lester, D. R., Plana, L. A., Garside, J. D., Painkras, E., Temple, S., et al. (2013). Overview of the spinnaker system architecture. IEEE Trans. Comput. 62, 2454-2467. doi: 10.1109/TC.2012.142

9. Gewaltig, M.-O., and Diesmann, M. (2007). NEST (NEural Simulation Tool). Scholarpedia 2:1430. doi: 10.4249/scholarpedia. 1430

10. Goodman, D., and Brette, R. (2008). Brian: a simulator for spiking neural networks in python. Front. Neuroinform. 2:5. doi: 10.3389/neuro.11.005.2008

11. Goodman, S. N., Fanelli, D., and Ioannidis, J. P. A. (2016). What does research reproducibility mean? Sci. Transl. Med. 8:341ps12. doi: 10.1126/scitranslmed.aaf5027

12. Gutzen, R., von Papen, M., Trensch, G., Quaglio, P., Grün, S., and Denker, M. (2018). Reproducible neural network simulations: statistical methods for model validation on the level of network activity data. Front. Neuroinform. 12:90. doi: 10.3389/fninf.2018.00090

13. Hines, M. L., and Carnevale, N. T. (1997). The NEURON simulation environment. Neural Comput. 9, 1179-1209. doi: 10.1162/neco.1997.9.6.1179

14. Hopkins, M., and Furber, S. (2015). Accuracy and efficiency in fixed-point neural ode solvers. Neural Comput. 27, 2148-2182. doi: 10.1162/NECO_a_00772

15. Lambert, J. D. (1992). Numerical Methods for Ordinary Differential Systems. New York, NY: Wiley.

16. Martin, R. C., and Coplien, J. O. (2009). Clean Code: A Handbook of Agile Software Craftsmanship. Upper Saddle River, NJ: Prentice Hall.

17. Martis, M. S. (2006). Validation of simulation based models: a theoretical outlook. Electron. J. Bus. Res. Methods 4, 39-46.

18. Patil, P., Peng, R. D., and Leek, J. (2016). A statistical definition for reproducibility and replicability. bioRxiv [Preprint]. doi: 10.1101/066803

19. Burov A.G., Agüero D. Implementation of the Principles of Innovative Entrepreneurship in the Field. Academy of Entrepreneurship Journal. 2019. Vol. 25. Issue 1S. pp. 1-5.

20. Shashkova, A., Verlaine, M., \& Kudryashova, E. (2020). On modifications to the constitution of the russian federation in 2020. Russian Law Journal, 8(1), 60-83. https://doi.org/10.17589/2309-8678-2020-8-1-60-83

21. Shashkova, A. (2019). Regulating principles of disclosure of information to shareholders under G20 / OECD principles. In Proceedings of the $33 \mathrm{rd}$ International Business Information Management Association Conference, IBIMA 2019: Education Excellence and Innovation Management through Vision 2020 (pp. 1931-1936).

22. Gennadievich, B. A. (2020). Machine learning and data mining activity results when using projectiles in different sports. International Journal of Advanced Trends in Computer Science and Engineering, 9(3), 3157-3160.

https://doi.org/10.30534/ijatcse/2020/103932020

23. Abramov, R. A. (2016). Regional economic policy based on industrial sector clustering in the context of sustainable development. Research Journal of Pharmaceutical, Biological and Chemical Sciences, 7(2), 2100-2106.

24. Abramov, R. A., Koshkin, A. P., Sokolov, M. S., \& Surilov, M. N. (2018). Transformation of the public administration system in the context of integration of the national innovation systems of the Union State. Espacios, 39(14).

25. Taran, S., \& Kolganova, I. (2018). Optimization of park plantings in the regions of Rostov-on-Don and Novocherkassk by introducing into gardening species of the genus ACER L. World Ecology Journal, 8(3), 56-70.

https://doi.org/https://doi.org/10.25726/NM.2019.31.46. 004

26. Tsembelev, M. (2018). Studies on the drought tolerance of species of the genus CELTIS L. for forest reclamation plantations. World Ecology Journal, 8(3), 71-85.

https://doi.org/https://doi.org/10.25726/NM.2019.44.92. 005

27. Amiripalli, S. S., Kollu, V. V. R., Jaidhan, B. J., Srinivasa Chakravarthi, L., \& Raju, V. A. (2020). Performance improvement model for airlines connectivity system using network science. International Journal of Advanced Trends in Computer Science and Engineering, 9(1), 789-792.

https://doi.org/10.30534/ijatcse/2020/113912020

28. Amirthalingam, G., \& Thangavel, H. (2019). Multi-biometric authentication using deep learning classifier for securing of healthcare data. International Journal of Advanced Trends in Computer Science and Engineering, 8(4), 1340-1347.

https://doi.org/10.30534/ijatcse/2019/48842019 